\title{
OVERRING PROPERTIES OF $G$-DOMAINS ${ }^{1}$
}

\author{
REVATI RAMASWAMY AND T. M. VISWANATHAN
}

\begin{abstract}
A commutative domain $R$ is called a strong $G$-domain if every overring between $R$ and the quotient field $K$ of $R$ is of the form $R[1 / t]$ for some nonzero element $t$ of $R$. After characterizing valuation rings which are strong $G$-domains, the authors show that $R$ is a strong $G$-domain if and only if it is a finite intersection of valuation rings each of which is a strong $G$ domain. Using some results of R. W. Gilmer, Jr., the authors identify the strong $G$-domains in the class of all Prüfer domains. They reprove via Krull domains the theorem characterizing Noetherian $G$-domains, a result first proved by Artin and Tate. The authors also raise some relevant questions on related overring properties of $G$-domains.
\end{abstract}

1. Introduction. An integral domain $R$ is said to be a $G$-domain if the quotient field $K$ of $R$ is a finitely generated ring over $R$. This is equivalent to saying that the quotient field $K$ is of the form $R[1 / t]$ for some nonzero element $t$ of $R$. As Kaplansky points out in [9, p. 13], very little is known about the structure of non-Noetherian $G$-domains. In this work, we characterize those $G$-domains $R$ which have the property that every overring of $R$ in the quotient field $K$ of $R$ is of the form $R[1 / t]$ for some $t \in R$. Such rings are designated strong $G$-domains. Theorem 3.5 shows that in contrast to the Noetherian case, the ideal theory of general strong $G$-domains is far from simple.

Our study of strong $G$-domains leads to a weaker notion, that of a locally pqr domain. A quotient ring $R^{\prime}$ of a domain $R$ with respect to a multiplicative set is called a principal quotient ring of $R$ if $R^{\prime}=R_{M}$ for a multiplicative set $M=\left\{1, t, \ldots, t^{n}, \ldots\right\}$ generated by a single element $t$ of $R^{\prime}$. In this case $R^{\prime}=R_{M}$ will also be denoted by $R_{t}$. With this definition, a domain $R$ is said to be locally pqr if for every prime ideal $P$ of $R$, the localization $R_{P}$ is a principal quotient ring of $R$. It is obvious that every strong $G$-domain is locally pqr and that every locally pqr domain is a $G$-domain. Examples show that the converse need not hold.

It is worth mentioning explicitly that Theorem 4.2 gives an elegant proof of the result characterizing Noetherian $G$-domains. Our line of study suggests some related notions about which questions are raised. It seems important enough to resolve these questions in order to gain insight into the structure of $G$-domains.

Received by the editors July 14, 1975.

AMS (MOS) subject classifications (1970). Primary 13F05, 13G05, 13B20, 13A15; Secondary $13 \mathrm{E} 05$.

Key words and phrases. G-domains, Noetherian G-domains, Prüfer domains, Krull domains, valuation rings, overrings, localization, spectrum of a commutative ring.

1 Work done while the second author was spending a semester at the Indian Institute of Technology, Kanpur in 1975 during an "afastamento do IMPA".

- American Mathematical Society 1976 
2. Locally pqr domains. In this section, we prove a key result for the locally pqr domains defined in $\$ 1$. This result is the finiteness of the number of maximal ideals of such domains. The following result will be repeatedly used.

PROPOSITION 2.1. Let $P$ be a prime ideal of a domain $R$ and $t$ an element of $R$ not in $P$. Then the following conditions are equivalent:

(1) $R_{P}=R_{t}$ is a principal quotient ring of $R$.

(2) For every element $b$ of $R$ not in $P$, the element $t$ belongs to the radical of $(b)$.

(3) $t$ belongs to every prime ideal $Q$ not contained in $P$.

Proof. (3) $\Rightarrow(2)$. Take $b \notin P$. The radical $(b)$ is the intersection of all the prime ideals containing $b$. From (3), it follows that $t$ belongs to this intersection.

(2) $\Rightarrow(1)$. By hypothesis $t \notin P$ which implies that the principal quotient ring $R_{t} \subseteq R_{P}$. If now, $x=a / b \in R_{P}$ with $a, b \in R$ and $b \notin P$, then (2) says that some positive power $t^{n} \in(b)$. Let $c \in R$ such that $t^{n}=b c$. Hence $x=a c / b c=a c / t^{n} \in R_{t}$. Thus $R_{P} \subseteq R_{t}$.

(1) $\Rightarrow$ (3). Let $R_{P}=R_{t}$ and $Q$ a prime ideal of $R$ not contained in $P$. If $q \in Q-P$, then $1 / q \in R_{P}$ and so $1 / q=a / t^{n}$ for some $a \in R$ and $n \geqslant 1$. This gives that $t^{n}=a q \in Q$. Thus $t \in Q$.

COROllary 2.2. A domain $R$ with a finite number of prime ideals is locally pqr.

COROLlaRY 2.3. If $R_{P}$ is a principal quotient ring of $R$, then $P$ is a G-ideal of $R$.

Proof. The corollary follows immediately from Proposition 2.1 and Theorem 19 of [9] by passage to the quotient $R / P$.

COROLlaRY 2.4. Every prime ideal of a locally pqr domain is a G-ideal.

REMARK 2.5. It may be seen from the following example that the converse of Corollary 2.3 may not hold. Take $R=Z$. Every maximal ideal $(p)$ of $Z$ is a $G$-ideal. However $Z_{(p)}$ is never a principal quotient ring of $Z$.

Proposition 2.6. Let $P$ be a prime ideal of a domain $R$. Suppose the localization $R_{P}$ is a principal quotient ring of $R$. Then the set $Y_{P}=\{Q \subseteq P: Q$ is a prime ideal of $R\}$ is an open subset of Spec $R$ with the Zariski topology.

Proof. By Proposition 2.1, the complement of $Y_{P}$ is precisely the set $V(t)$ of all prime ideals containing $t$ which is a closed subset of Spec $R$.

THEOREM 2.7. Every locally pqr domain is a semilocal ring, that is, has only a finite number of maximal ideals.

Proof. Let $\left\{M_{i}\right\}_{i \in I}$ be the family of maximal ideals of $R$ and $\left\{t_{i}\right\}_{i \in I}$ a family of elements of $R$ defining the $R_{M_{i}}$ as principal quotient rings $R_{t_{i}}$. By Proposition 2.6 each $Y_{M_{i}}$ is an open subset of Spec $R$. Now, $\cup_{i \in I} Y_{M_{i}}$ is an open covering of the compact space Spec $R$. Hence it admits a finite subcovering say $Y_{M_{1}} \cup Y_{M_{2}} \cup \cdots \cup Y_{M_{r}}$. It is obvious then that $M_{1}, M_{2}, \ldots, M_{r}$ are all the maximal ideals of $R$.

REMARK 2.8. Notice that the conclusion of Theorem 2.7 continues to be valid in a domain $R$ in which all localizations with respect to maximal ideals are principal quotient rings. However a semilocal domain need not be locally 
pqr as may be seen by Example 5.1 of a valuation domain which is not even a $G$-domain.

Proposition 2.9. A locally pqr domain satisfies the d.c.c. on prime ideals.

Proof. Let $P=P_{0} \supset P_{1} \supset \cdots \supset P_{m} \cdots$ be an infinite strictly descending chain of prime ideals of $R$ and consider the prime ideal $Q=\cap_{i=0}^{\infty} P_{i}$. Since the chain is not finite, $Q \varsubsetneqq P_{i} \quad \forall i \geqslant 0$. If $R_{Q}=R_{t}$ for some $t \in R$, then $t \in P_{i}$ for every $i$ by Proposition 2.1. Hence $t \in Q$, their intersection, contradicting the fact that $t \notin Q$. This proves the proposition.

We will later need the following stability result.

PROPOSITION 2.10. Any quotient ring of a locally pqr domain is again locally pqr.

Proof. Let $R^{\prime}=R_{S}$ be a quotient ring of a locally pqr domain $R$ with respect to a multiplicative set $S$ of $R$. Let $P^{\prime}=P^{e}$ be an extension to $R^{\prime}$ of a prime ideal $P$ of $R$. By (4.3) of [7], we get $R_{P^{\prime}}^{\prime}=R_{P}$ and by hypothesis $R_{P}$ is a principal quotient ring $R_{t}$ of $R$. Hence $R_{P^{\prime}}^{\prime}=R_{t}^{\prime}$ is a principal quotient ring of $R^{\prime}$.

The following result on locally pqr domains is somewhat curious.

PROPOSITION 2.11. Let $f$ be any element of a locally pqr domain. Then there are only a finite number of prime ideals of $R$ maximal with respect to not containing $f$.

Proof. Apply the reasoning of Theorem 2.7 to the compact set $X_{f}$ $=\{P \in \operatorname{Spec} R: f \notin P\}$.

3. Strong $G$-domains. The class of strong $G$-domains defined in $\S 1$ appears to be the nicest class of $G$-domains. It will be clear later that this class includes all Noetherian integrally closed $G$-domains. Before we take up the ideal theory of these domains, we need to establish some preliminary results.

LEMMA 3.1. Let $\left\{t_{i}\right\}_{i=1}^{i=n}$ be a family of nonzero elements of a domain $R$ such that $t=t_{1}+t_{2}+\cdots+t_{n} \neq 0$. Then $R_{t_{1}} \cap R_{t_{2}} \cap \cdots \cap R_{t_{n}} \subseteq R_{t}$.

Proof. Let $0 \neq y \in \cap_{i=1}^{n} R_{t_{i}}$. Hence there exist elements $a_{1}, a_{2}, \ldots, a_{n}$ in $R$ and positive integers $m_{1}, \ldots, m_{n}$ such that $y=a_{1} / t_{1}^{m_{1}}=a_{2} / t_{2}^{m_{2}}=\cdots$ $=a_{n} / t_{n}^{m_{n}}$. Thus $a_{i}=y t_{i}^{m_{i}}, \quad i=1,2, \ldots, n$. Let $m=m_{1}+m_{2}+\cdots+m_{n}$. Then each term of the multinomial $y\left(t_{1}+t_{2}+\cdots+t_{n}\right)^{m}$ is in $R$ and so $y\left(t_{1}+\cdots+t_{n}\right)^{m}=r \in R$. Since $t=\left(t_{1}+\cdots+t_{n}\right) \neq 0$ and $y \neq 0$, we get $y \in R_{t}$.

LEMMA 3.2. Let $P_{1}, \ldots, P_{n}$ be a family of mutually incomparable prime ideals of $R$. Suppose each $R_{P_{i}}$ is a principal quotient ring of $R$. Then so is $R^{\prime}$ $=\bigcap_{i=1}^{n} R_{P_{i}}$.

Proof. By Proposition 2.1, there exist elements $t_{1}, t_{2}, \ldots, t_{n}$ of $R$ such that $t_{i} \notin P_{i}$ and $t_{i} \in Q$ for every $Q \nsubseteq P_{i}$. In particular $t_{i} \in P_{j} \quad \forall j \neq i$. Hence $0 \neq t=t_{1}+\cdots+t_{n} \notin \cup_{i=1}^{n} P_{i}$. Let $S$ be the complement of $\cup_{i=1}^{n} P_{i}$. Hence $R_{S}=\bigcap_{i=1}^{n} R_{P_{i}}$ by (4.4) of [7]. Since $t \in S$, clearly $R_{t} \subseteq R_{S}$. By Lemma 3.1, $R_{S} \subseteq R_{t}$ and so $R_{t}=R_{S}=\bigcap_{i=1}^{n} R_{P_{i}}$.

Our next result identifies the strong $G$-domains among valuation rings. 
THEOREM 3.3. Let $V$ be a valuation ring. Then the following conditions are equivalent:

(1) $V$ is a strong G-domain.

(2) $V$ is a locally pqr domain.

(3) Every prime ideal of $V$ is a $G$-ideal.

(4) The family of prime ideals of $V$ is well ordered with respect to inclusion.

Proof. (1) $\Rightarrow(2)$ is trivial. (2) $\Rightarrow(3)$ is Corollary 2.4 .

$(3) \Rightarrow(4)$. Let $\mathscr{F}$ be any nonempty family of prime ideals of $V$. Then $Q=\cap \mathscr{F}$ is a prime ideal of $V$. If $Q \in \mathscr{F}$, then $Q$ is the first element of $\mathscr{F}$ with respect to inclusion and we are done with the proof. We shall show that the assumption that $Q \notin \mathcal{F}$ gives rise to a contradiction. First of all $Q \subseteq P_{i}$ for every $P_{i} \in F_{\text {. By }}(3), V / Q$ is a valuation $G$-domain and so the intersection of all the nonzero primes of $V / Q$ is a nonzero prime $Q^{\prime} / Q$. Hence $Q \subseteq Q^{\prime} \subseteq P_{i}$ for every prime $P_{i} \in \mathcal{F}$, showing that $Q=\cap \mathcal{F}=Q^{\prime}$, a contradiction.

(4) $\Rightarrow(1)$. Let $V^{\prime}=V_{P}$ be any overring of $V$. If $P$ is the maximal ideal of $V$, then $V=V_{P}$. If $P$ is not maximal, let $Q$ be a prime ideal which is minimal among all primes strictly containing $P$. $Q$ exists by (4). Pick $t \in Q \backslash P$. Then $V_{P}=V_{t}$ by Proposition 2.1 .

We next consider a finite intersection of valuation $G$-domains.

THEOREM 3.4. Let $V_{1}, V_{2}, \ldots, V_{n}$ be a finite number of incomparable valuation strong G-domains. Then $R=\cap_{i=1}^{n} V_{i}$ is a strong G-domain.

Proof. We break down the proof into five steps.

Step 1. $R$ is a Prüfer domain with a finite number of maximal ideals $M_{1}, M_{2}, \ldots, M_{n}$ and indeed $V_{i}=R_{M_{i}}$ for every $i=1,2, \ldots, n$. This wellknown fact is (11.12) of [4].

Step 2. Any family of mutually incomparable prime ideals of $R$ is finite.

If $\mathscr{F}=\left\{P_{i}\right\}_{i \in I}$ be the given family, let $P_{1} \in \mathscr{F}$ be contained in $M_{1}$ say. If $P_{2} \neq P_{1}$ is another element of $\mathscr{F}$, then $P_{2}$ is incomparable to $P_{1}$ and since the ideals of the valuation ring $R_{M_{1}}$ are totally ordered with respect to inclusion, we cannot have $P_{2} \subseteq M_{1}$. Hence $P_{2} \subseteq M_{2}$ say. Continuing in this way, we can get at most $n$ distinct elements of $\mathscr{F}$ and $\mathscr{F}$ will be a finite set.

Step 3. Any nonempty family of prime ideals of $R$ has minimal elements and these are finite in number.

Let $\mathscr{F}$ be such a family and $Q \in \mathscr{F}$. If $Q \subseteq M_{1}$, then $R_{Q}$ is an overring of the strong $G$-domain $R_{M_{1}}$. Hence $R_{Q}$ is also a valuation strong $G$-domain. Thus $\mathscr{F} \cap \operatorname{Spec} R_{Q}$ has a first element by Theorem 3.3, which will be a minimal element of $\mathscr{F}$. Thus $\mathscr{F}$ has minimal elements. Their finiteness is a consequence of Step 2.

Step 4. If $R^{\prime}$ is an overring of $R$, then there exist a finite number of primes $P_{1}, P_{2}, \ldots, P_{m}$ of $R$ such that $R^{\prime}=\cap_{i=1}^{m} R_{P_{i}}$.

Let $\left\{A_{i}\right\}_{i \in I}$ be the family of maximal ideals of $R^{\prime}$. Let $B_{i}=A_{i} \cap R$. By Theorem 1 of [6], we have $B_{i} R^{\prime}=A_{i}$ and so $\left\{B_{i}\right\}_{i \in I}$ is a family of mutually incomparable prime ideals of $R$. By Step 2, this family is finite. Rename the $B_{i}$ as $P_{1}, P_{2}, \ldots, P_{m}$. Then using maximality and Theorem 1 of [6], we get $R^{\prime}=\bigcap_{i=1}^{m} R_{P_{i}}$. 
Step 5. $R^{\prime}$ is a principal quotient ring of $R$. By Lemma 3.2, it is enough to prove that each $R_{P_{i}}$ is a principal quotient ring of $R$. Let us for example prove that $R_{P_{1}}=R_{t_{1}}$ for some $t_{1} \in R$. For this, consider the set $\delta_{1}=\left\{Q \nsubseteq P_{1}: Q\right.$ is a prime ideal of $R$ \}. Since we may assume that $R$ is not local, $\delta_{1}$ is not empty. Let $Q_{1}, Q_{2}, \ldots, Q_{r}$ be the minimal elements of $\delta_{1}$. Choose an element $t_{1} \notin P_{1}$ but belonging to every $Q_{i}, i=1,2, \ldots, r$. Clearly $R_{P_{1}}=R_{t_{1}}$.

It is an easy matter now to prove our main result.

THEOREM 3.5. The following conditions are equivalent for an integral domain $R$ :

(1) $R$ is a strong G-domain.

(2) $R$ is a Prüfer domain which is locally pqr.

(3) $R$ is a finite intersection of valuation rings each of which is a strong $G$ domain.

Proof. (1) $\Rightarrow(2) . R$ is clearly locally pqr and $R$ has the QR property defined in [6]. In particular every valuation overring of $R$ is a quotient ring of $R$. Hence $R$ is a Prüfer domain.

(2) $\Rightarrow(3)$. By Theorem $2.7, R$ has only a finite number of maximal ideals $M_{1}, M_{2}, \ldots, M_{n}$ and each $R_{M_{i}}$ is a valuation ring and $R=\bigcap_{i=1}^{n} R_{M_{i}}$. To show that each $R_{M_{i}}$ is a strong $G$-domain, observe that a prime ideal of $R_{M_{i}}$ is of the form $P^{e}$ with $P$ a prime ideal of $R$. Then by 4.3 of [7], $\left(R_{M_{i}}\right)_{P e}=R_{P}$. Since $R_{P}=R_{t}$ by (2), we get that $\left(R_{M_{i}}\right)_{P e}$ is a principal quotient ring of $R_{M_{i}}$. (3) $\Rightarrow(1)$ is Theorem 3.4 .

COROLlaRY 3.6. A strong G-domain $R$ is integrally closed and has only a finite number of minimal and maximal prime ideals.

Proof. This follows from the fact that $R$ is Prüfer and from Step 3 of Theorem 3.4.

4. Noetherian $G$-domains. In this section, we shall obtain an elegant proof of the theorem characterizing Noetherian $G$-domains, first proved by Artin and Tate in [2]. See also [9, p. 107]. Our approach to this theorem is via Krull domains.

THEOREM 4.1. The following conditions are equivalent for a Krull domain $R$ :

(1) $R$ is a G-domain.

(2) $R$ is a PID with a finite number of prime ideals.

(3) $R$ is a strong G-domain.

(4) $R$ is locally $p q r$.

Proof. (1) $\Rightarrow(2)$. Since $R$ is a $G$-domain, by Proposition 2.1, there exists an element $t$ belonging to every nonzero prime $P$ of $R$. But in a Krull domain, every nonzero element belongs only to a finite number of minimal prime ideals (p. 82 of [9]). This means that $R$ has only a finite number of minimal prime ideals. Hence $R$ is Dedekind and a PID.

(2) $\Rightarrow(3)$. If $P_{1}, P_{2}, \ldots, P_{m}$ are the nonzero primes of $R$, then $R=\bigcap_{i=1}^{m} R_{P_{i}}$ and each $R_{P_{i}}$ is a rank one valuation ring. Hence by Theorem 3.4, $R$ is a strong $G$-domain.

(3) $\Rightarrow(4)$ and (4) $\Rightarrow(1)$ are trivial. 
We can now take up the Noetherian case.

THEOREM 4.2. Let $R$ be a Noetherian domain. Then the following conditions are equivalent:

(1) $R$ is a G-domain.

(2) $R$ is locally pqr.

(3) $R$ has only a finite number of nonzero prime ideals each of which is maximal.

(4) The integral closure of $R$ is a principal ideal domain with a finite number of prime ideals.

Proof. (1) $\Rightarrow(4)$. By the Mori-Nagata integral closure theorem (Theorem 4.3 of [5]), the integral closure $\bar{R}$ of $R$ is a Krull domain. Being an overring of $R$, it is also a $G$-domain. Hence by Theorem $4.1, \bar{R}$ is a PID with a finite number of primes.

$(4) \Rightarrow(3)$. By (4), $\bar{R}$ has only a finite number of nonzero primes, each of which is maximal. Hence the same is true for $R$ (by Theorem 3 and its consequences on pp. 257-259 of Vol. I of [11]).

(3) $\Rightarrow(2)$ is Corollary 2.2 .

(2) $\Rightarrow(1)$ is trivial.

Example 5.4 below shows that a Noetherian $G$-domain need not be a strong $G$-domain.

\section{Examples.}

EXAMPLE 5.1. A valuation ring $V$ need not be a $G$-domain. This fact is well known. In this case the family of proper convex subgroups of the value group $V$ cannot have a maximal element with respect to inclusion. An explicit example is given by a valuation ring whose value group is the Hahn group $\mathcal{H}_{i \in I} Z_{i}$, where $I$ is the ordered set $\{1,2,3 \cdots\}$ and each $Z_{i}$ is the ordered group of integers. For this construction and other details, see [10].

EXAMPLE 5.2. A valuation $G$-domain $V$ need not be a strong $G$-domain. By Theorem 3.3, it is enough to consider a valuation $G$-domain whose prime ideals are not well ordered with respect to inclusion. This is equivalent to saying that the family of convex subgroups of the value group $G$ is not antiwell-ordered with respect to inclusion. An explicit example of such an ordered group is given by the lexicographic product $\mathcal{H}_{i \in I \cup\{w\}} Z_{i}$ where $I=\{1,2, \ldots\}$ has the usual order and $w>i \quad \forall i \in I$ and where each $Z_{i}$ is the ordered group of integers.

EXAMPLE 5.3. A strong G-domain may have any rank. Let $I$ be an anti-wellordered set of a given order type. Then any valuation ring $V$ whose value group is isomorphic to the Hahn group $\mathcal{H}_{i \in I} Z_{i}$ constructed as above has rank equal to that of $I$. However $V$ is a strong $G$-domain.

EXAMPLE 5.4. A Noetherian $G$-domain need not be a strong $G$-domain. Let $k$ be a field and $K=k((X))$ the field of formal power series over $k$. Let $\bar{R}$ be the discrete valuation ring defined by

$$
\bar{R}=\left\{\sum_{i=0}^{\infty} a_{i} X^{i}: a_{i} \in k\right\}
$$

and let 


$$
R=\left\{\sum_{i=0}^{\infty} a_{i} X^{i}: a_{i} \in k, a_{1}=0\right\}
$$

be the ring obtained from $\bar{R}$ by "dropping" $X$. It is easily checked that $R$ is a Noetherian domain with a unique nonzero prime ideal $P=\left(X^{2}, X^{3}\right) . R$ is thus a Noetherian $G$-domain. But $R$ is not integrally closed and in fact the integral closure of $R$ is $\bar{R}$. Notice that $\bar{R}=R[X]$ cannot be a quotient ring of $R$, since the quotient field $K$ is the only quotient ring of $R$. Thus $R$ is not a strong $G$-domain.

EXAMPLE 5.5. A locally pqr domain need not be a strong G-domain. From this, one may take the above example. The following example is also interesting from another point of view. Take $R=Z[\sqrt{5}]$. Then $P=(1+\sqrt{5}, 1-\sqrt{5})$ is a prime ideal of $R$ and in fact $P$ is the only prime ideal of $R$ which is not invertible in $R$. Let $R^{\prime}=R_{P}$. Then $R^{\prime}$ is a Noetherian locally pqr domain which is not integrally closed. Thus $R^{\prime}$ is not a strong $G$-domain.

6. Some open questions. We now indicate some of the questions which arise in a natural way in our study. It is imperative to answer some of these questions in order to gain further insight into the structure of $G$-domains. Some of these answers will also test the strength of our theory of strong $G$ domains. And most important of all, they will provide more examples for the theory and clues for further development.

The following concept is very natural in this context: we say that the domain $R$ has the finite overring property if every overring of $R$ is a finitely generated ring over $R$.

It is easy now to prove the following:

Proposition 6.1. If the domain $R$ has the finite overring property, then $R$ is locally pqr.

Proof. Let $P$ be a prime ideal of $R$ and let $R_{P}=R\left[a_{1} / b_{1}, \ldots, a_{n} / b_{n}\right]$ with $a_{i}, b_{i} \in R$. Write $a_{i} / b_{i}=r_{i} / t_{i}$ with $t_{i} \notin P$. If $t=t_{1} \cdot t_{2} \cdots t_{n}$, then $R_{P} \subseteq R_{t}$ $\subseteq R_{P}$, whence equality. Thus each $R_{P}$ is a principal quotient ring of $R$.

This leads to our first question which the authors are unable to solve.

Question 6.2. If $R$ has the finite overring property, is it true that $R$ is a strong $G$-domain?

Question 6.3. Let $R$ be an integrally closed domain with the property that every valuation overring of $R$ is a finitely generated ring over $R$. Does $R$ have the finite overring property?

Question 6.4. Is every overring of a locally pqr domain locally pqr?

The authors hope that the answers to these questions will be stimulating further interest on $G$-domains. In this context we draw the attention of the reader to the work of Adams in [1] and of Guerindon in [8]. The authors wish to thank Professor Robert Gilmer, Jr. for supplying them with copies of these two works and of [6]. Thanks are also due to Professor Manfred Knebusch for permitting the typing of the manuscript in the Universität Regensburg and to Dr. G. V. Krishna Rao for many discussions when the work was in progress.

ADDED IN PROOF. Revati Ramaswamy now has an answer to Question 6.3. T. M. Viswanathan has answered Question 6.2 in the negative. 


\section{REFERENCES}

1. John Conway Adams, Rings with a finitely generated total quotient ring, Canad. Math. Bull. 17 (1974), 1-4.

2. Emil Artin and John T. Tate, A note on finite ring extensions, J. Math. Soc. Japan 3 (1951), 74-77. MR 13, 427.

3. M. F. Atiyah and I. G. MacDonald, Introduction to commutative algebra, Addison-Wesley, Reading, Mass., 1969. MR 39 \#4129.

4. O. Endler, Valuation theory, Universitext, Springer-Verlag, New York and Heidelberg, 1972. MR 50 \# 9847.

5. Robert M. Fossum, The divisor class group of a Krull domain, Ergebnisse der Mathematik und ihrer Grenzgebiete, vol. 74, Springer-Verlag, Berlin, 1973.

6. R. W. Gilmer, Jr., Overrings of Prüfer domains, J. Algebra 4 (1966), 331-340. MR 34 \#2609.

7. _ Multiplicative ideal theory, Queen's Papers in Pure and Appl. Math., no. 12, Queen's University, Kingston, Ont., 1968. MR 37 \#198.

8. J. Guerindon, Anneaux de Goldman (to appear).

9. I. Kaplansky, Commutative rings, Allyn and Bacon, Boston, Mass., 1970. MR 40 \#234.

10. P. Ribenboim, Théorie des valuations, 2ième éd., multigraphiée, Séminaire de Mathématiques Supérieures, no. 9 (Été, 1964), Les Presses de l'Université de Montréal, Montréal, Qué., 1968. MR 40 \#2670.

11. O. Zariski and P. Samuel, Commutative algebra. Vols. 1, 2, Univ. Ser. in Higher Math., Van Nostrand, Princeton, N. J., 1958, 1960. MR 19, 833; 22 \#11006.

Department of Mathematics, Indian Institute of Technology, KanPur 208016, India

Instituto de Matemática Pura e Aplicada, Rua luis de Camões, 68, 20.000 Rio de JANEIRo, RJ, BRAZIL 